Original Research Paper

\title{
Determination of Abscisic Acid and its Relationship to Drought Stress Based on Cowpea Varieties with Different Capability of Drought Resistance
}

\author{
${ }^{1}$ Wei Lei, ${ }^{1}$ Shian Huang, ${ }^{2}$ Shaohu Tang, ${ }^{3}$ Xiaorong Shui and ${ }^{1}$ Can Chen \\ ${ }^{1}$ Laboratory of Cardiovascular Diseases, Guangdong Medical College, Zhanjiang 524000, China \\ ${ }^{2}$ Key Laboratory of Eco-Environments in Three Gorges Reservoir Region (Ministry of Education), \\ College of Life Science, Southwest University, Chongqing 400715, China \\ ${ }^{3}$ Laboratory of Vascular Surgery, Guangdong Medical College, Zhanjiang 524001, China
}

\author{
Article history \\ Received: 04-12-2015 \\ Revised: 28-03-2016 \\ Accepted: 29-03-2016 \\ Corresponding Author: \\ Wei Lei \\ Laboratory of Cardiovascular \\ Diseases, Guangdong Medical \\ College, Zhanjiang 524000, \\ China \\ Xiaorong Shui \\ Laboratory of Vascular \\ Surgery, Guangdong Medical \\ College, Zhanjiang 524001, \\ China \\ Email: yangtse2006@yahoo.com \\ shuixiaor@126.com
}

\begin{abstract}
Cowpea (Vigna unguiculata L.) is one of the most important grain legume supplying necessarily dietary proteins. Although its can tolerate drought environment, still the severe condition can limit the growth and development of this plant. Abscisic acid (ABA) has a crucial function in stress physiology, but little information is available for methods describing ABA determination in cowpea and its relationship to drought stress based on cowpea varieties with different capability of drought resistance. Here, an effectual approach based on high-performance liquid chromatography (HPLC) method was established to separate and determine the endogenous abscisic acid in cowpea and further the cowpea varieties with different capability of drought resistance were used to explore relationships between ABA content and drought stress. The results potentially provided useful reference for the cultivation of cowpea crop. Results-This method showed good liner regression $\left(R^{2}=0.9998\right)$, coupled with high stability, precision and repeatability. The endogenous ABA decreased under drought treatment and the drought sensitive line had higher ABA content than drought resistant one, suggesting ABA performs a vital function in cowpea's response and resistance to drought stress. The studies potentially provided useful reference for the cultivation and breeding of cowpea crop.
\end{abstract}

Keywords: High-performance liquid chromatography, Cowpea, Abscisic acid, Drought

\section{Introduction}

Cowpea (Vigna unguiculata L.) is one of the most important drought-tolerant grain legume supplying necessarily dietary proteins, in particular for the vegetarian population of developing countries (Popelka et al., 2004; Ramakrishnan et al., 2005). Due to its super droughttolerant ability, it is largely cultivated as an indispensable food or forage crop in the semi-arid tropic areas of Africa, Asia, Latin America, where the yield of most crop plants has been compromised by the abnormally high temperature and low level of water supplying (Paul et al., 2011). Cowpea possesses several excellent nutritional qualities including high content of proteins, high calories, minerals and vitamins. It is an exclusively essential rural diet and animal feed, as well as a popular vegetable in the rest of the world. Besides, its biological nitrogen fixation property grants it an even more vital niche in enhancing soil fertility and improving soil structure in the era of widely appealing for organic food and environmentally friendly agriculture (Bressani, 1985; Martins et al., 2003), therefore, world demand for cowpea is increasing gradually. However, its production has, to some extent, been affected by drought stress, which has already become a hotspot topic in the field of agriculture (Bayuelo-Jimenez et al., 2002; Chen et al., 2007). Although cowpea can tolerate drought environment, still the severe condition can limit the growth and development of this plant (Munns, 1993). Abscisic acid (ABA) has a crucial function in stress physiology, but little information is available for methods describing ABA determination in cowpea and its relationship to drought stress based on cowpea varieties with different capability of drought resistance. 
$\mathrm{ABA}$, a type of sesquiterpenoid phytohormone, is commonly synthesized in all vascular plants, mosses and algae (Schwartz et al., 2003; Rai et al., 2011). Generally, $\mathrm{ABA}$ is maintained at relatively high level under the normal condition, but it will decrease significantly when plant is suffered from the environmental stresses (Xiong and Zhu, 2003; Sandhu et al., 2011). ABA acts as a key signal molecule in the regulation and induction of the plant physiological responses to osmotic stress which is induced by salt, drought and cold tolerance (Rai et al., 2011; Tuteja, 2007; Fujita et al., 2011). In particular when plant encounters drought environment, $\mathrm{ABA}$, with the content decreasing, stimulates stomatal closure and leaves wilt to mediate plant defense reaction (Bano and Yasmeen, 2010; Majeed et al., 2011; Fukao et al., 2011). These highlighted the importance of ABA in the plant's response to drought stress and great attention has been focused on the role of ABA in the stress response. Some analytical methods have been reported to determine this endogenous phytohormone, such as bioassays (Boerjan et al., 1992), gas chromatography (GC) (Du et al., 2000), enzyme linked immunosorbent assay (ELISA) (Gao et al., 2006; Ferreira et al., 2006), spectrometry, electrochemical technique (Carretero and Cruces-Blanco, 2004; Li et al., 2005) and high-performance liquid chromatography (HPLC) (Dobrev et al., 2005), in which HPLC has the highest sensitivity, the most specificity and the best repeatability in comparison with other methods mentioned, thus it is used extensively to determine ABA content in some plants such as soybean, Arabidopsis thaliana and Glycyrrhiza uralensis etc. (Ciha et al., 1977; Ross et al., 2004; Xu et al., 2010). In the present study, an effectual approach based on HPLC method was established to separate and determine the endogenous abscisic acid in cowpea and further relationships were explored between ABA content and drought stress. The results potentially provided useful reference for the cultivation and breeding of cowpea crop.

\section{Materials and Methods}

\section{Chemicals and Reagents}

HPLC-grade acetonitrile and methanol was obtained from Fisher Chemical, USA. Phosphoric acid, ethyl acetate and hydrochloric acid were of analytical grade and were purchased from Guahua Chemical Ltd. (China). Water for HPLC analysis was purified using Milli-Q water system (Millipore Corporate, France). ABA was obtained from Sanland Chemical Co., Ltd (Los Angeles, USA). All of the chromatographic grade solutions were stored at $4^{\circ} \mathrm{C}$ in darkness and then the HPLC mobile phase was prepared fresh at a time, filtered through a $0.45 \mu \mathrm{m}$ membrane filter.

\section{Plant Material and Drought Stress Treatment}

Cowpea materials used in current study were originated from Africa and contained two varieties: Dan lla (drought resistance variety) and TVu7778 (drought sensitive variety). Cowpea seeds were first surface sterilized in $1 \% \mathrm{NaClO}$ for $10 \mathrm{~min}$, then rinsed thrice in the sterile water. The sterilized seeds were set into earthen pots in a greenhouse at $30 \pm 2^{\circ} \mathrm{C}$. Twenty-two days later, sixteen uniform seedlings from each variety were divided into two groups, each having eight seedlings. After flood irrigation, the control groups for each variety were irrigated lightly every three days with soil moisture of more than $14.5 \%$; on the other hand, the experimental groups, was withheld irrigation for one month to maintain moisture around 5\% (Fig. 1). The soil moisture was measured by soil temperature and humidity speed measuring device purchased from Handan Electronic Technology Limited Company (China).

\section{Sample Preparation}

About $1.0 \mathrm{~g}$ of fresh cowpea leaves were collected and put into liquid nitrogen, followed by grinding into powder and then the powder was extracted in $15 \mathrm{~mL}$ of methanol $(80 \% \mathrm{v} / \mathrm{v})$ for $12 \mathrm{~h}$ at $4{ }^{\circ} \mathrm{C}$. After air pump filtration, the filtrate was obtained; the filtrate residue was extracted for another $2 \mathrm{~h}$ with subsequent pumping filtration, two filtrates were combined together. Three steps were carried out, including acidifying with hydrochloric acid, extracting with ethyl acetate thrice and air drying by vacuum reduced pressure concentration, the final volume of extrate was made up to $10 \mathrm{~mL}$ with addition of methanol. At last, the samples were filtrated through the $0.45 \mu \mathrm{m}$ membrane filter before being injected into HPCL system (Ma et al., 2011).

\section{Apparatus and Chromatographic Conditions}

Separation and determination of ABA were performed with HPLC system (Waters 2695, miford, MA, USA), which consisted of a quaternary pump, an ultraviolet detector (ELSD, Waters 2487) and Waters Empower software. Chromatographic condition was slightly modified from the previous report (Xu et al., 2010). Separation was carried out on a Waters Symmetry C18 column $(4.6 \times 250 \mathrm{~mm}, 5 \mu \mathrm{m})$ and this process was maintained at $25^{\circ} \mathrm{C}$ with a gradient elution at the flow rate of $0.6 \mathrm{~mL} \mathrm{~min}^{-1}$. The mobile phase was composed of acetonitrile (solvent A) and phosphoric acid solution $(0.1 \% \mathrm{v} / \mathrm{v})$ (solvent B). The gradient elution program was: $20 \% \mathrm{~A}$ and $80 \% \mathrm{~B}(0 \mathrm{~min}), 25 \% \mathrm{~A}$ and $75 \% \mathrm{~B}(5$ $\mathrm{min}), 30 \% \mathrm{~A}$ and $70 \% \mathrm{~B}(8 \mathrm{~min}), 35 \% \mathrm{~A}$ and $65 \% \mathrm{~B}(15$ $\mathrm{min}), 45 \% \mathrm{~A}$ and $55 \% \mathrm{~B}(25 \mathrm{~min})$. The target component was quantified by the peak areas at the maximum wavelength of $260 \mathrm{~nm}$. 


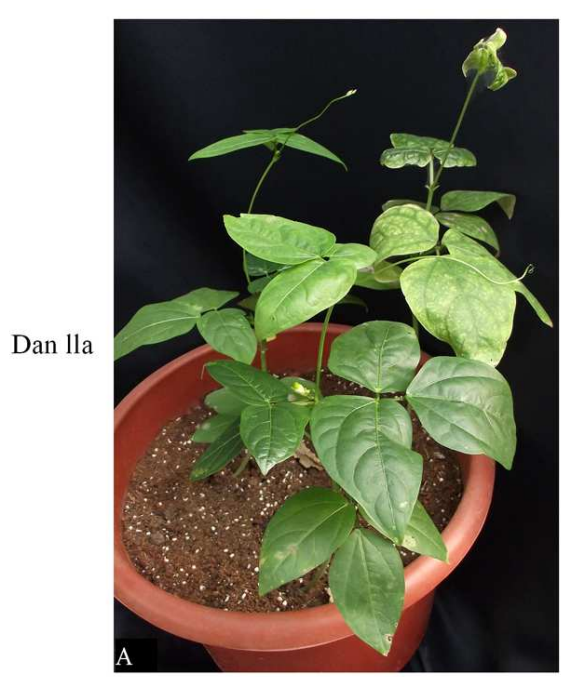

Control

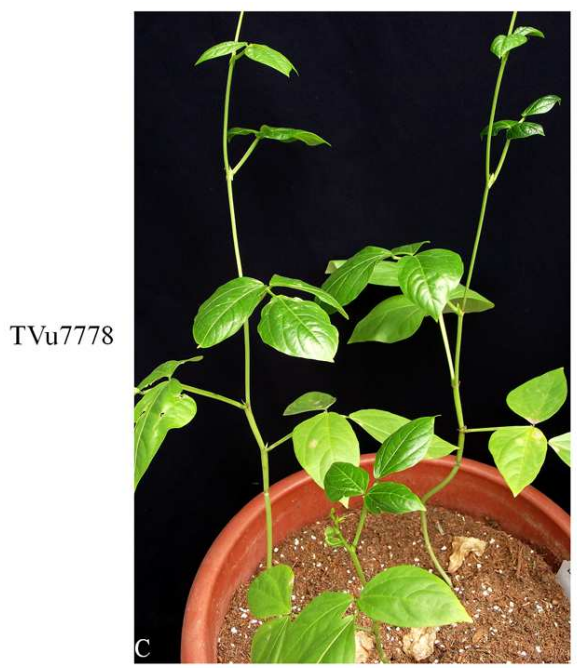

Control

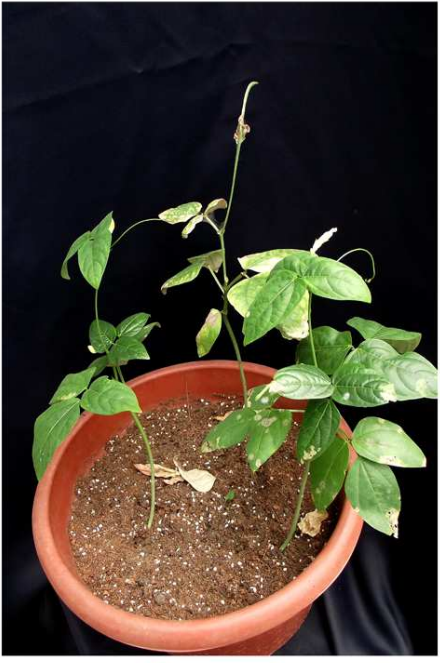

Drought

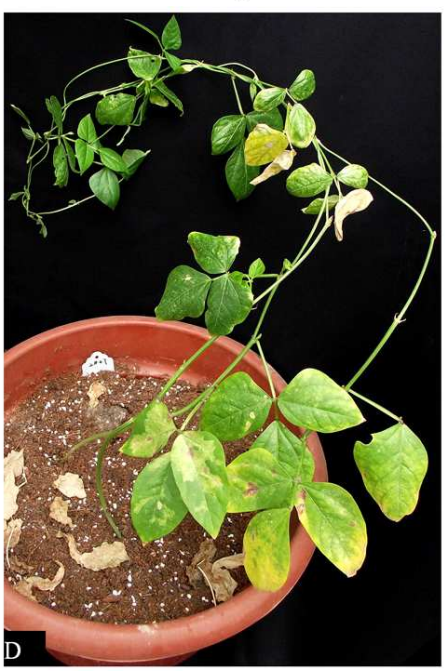

Drought

Fig. 1. Preparation of cowpea samples. A. the control group of Dan lla (moisture $15.64 \%, \mathrm{n}=8$ ); B. the treatment group of Dan 1 la (moisture $6.39 \%, n=8$ ); C. the control group of TVu7778 (moisture $11.91 \%, n=8$ ); D: The treatment group of TVu7778 (moisture $6.19 \%, \mathrm{n}=8$ ). Cowpea plants grown in soil were exposed to drought stress achieved by withholding irrigation for one month. The control plants were irrigated normally. A representative photograph is displayed

\section{Preparation of Standard Solutions and Calibration Curve}

Standard solutions of ABA were directly prepared in methanol. The assay linearity was determined for five concentrations of $33.6,16.8,6.73,3.36$ and $1.68 \mu \mathrm{g}$ $\mathrm{mL}^{-1}$, respectively. The calibration curve was constructed by the LC software and statistic analyses and the liner regression equation was obtained by plotting the peak areas vs. the nominal concentrations of ABA standard solutions. The linearity relationship was demonstrated by a correlation coefficient $\left(R^{2}\right)$ higher than 0.999 . LOD and LOQ were defined as the quantity of compound required for signal-to-noise ratios of $3: 1$ and 10:1, respectively.

\section{Method Validation}

The precision of this method was determined by repeated measurements of five times, the stability tests were carried out by analyzing ABA samples on 0 , $2,4,6,8 \mathrm{~h}$ and the repeatability was assessed by five independently prepared samples of ABA, with all indices calculated as R.S.D. In order to evaluate recovery of $\mathrm{ABA}$ in the current method, known amount of ABA standard solutions were injected at different levels of $33.6,16.8,6.72,3.36,1.68 \mu \mathrm{g} \mathrm{mL}^{-1}$ based on the described procedure and calculated according to the following formula: Recovery rate (\%) $=($ determined amount after spiking-original amount in sample)/spiked amount $\times 100 \%$. 


\section{Results}

\section{Optimization of Chromatographic Conditions}

The desired ABA compound from the samples was identified by comparing the retention time and UV spectra with that of the authentic standard. The remarkable agreement between the determined compound from the samples of cowpea and standard material suggested that the endogenous $\mathrm{ABA}$ was separated by gradient elution in about $21 \mathrm{~min}$ and determined accurately without interference from other components in the methanol extract of cowpea. As shown in Fig. 2, the typical chromatogram under the proposed conditions was recorded about standard $\mathrm{ABA}$ (Fig. 2A) and methanol extract of cowpea (Fig. 2B).

\section{Validation of Analytical Method}

Some indices, including $R^{2}$, LOD, LOQ, R.S.D, were used to evaluate precision, repeatability and stability of this method (Table 1). Both LOD and LOQ were examined to estimate the sensitivity of UV detector in the present study. The assay linearity was confirmed based on five gradient concentration of standard solutions and the regression equation coupled with calibration curve showed good fitness $\left(R^{2}=\right.$ 0.9998) (Fig. 3).
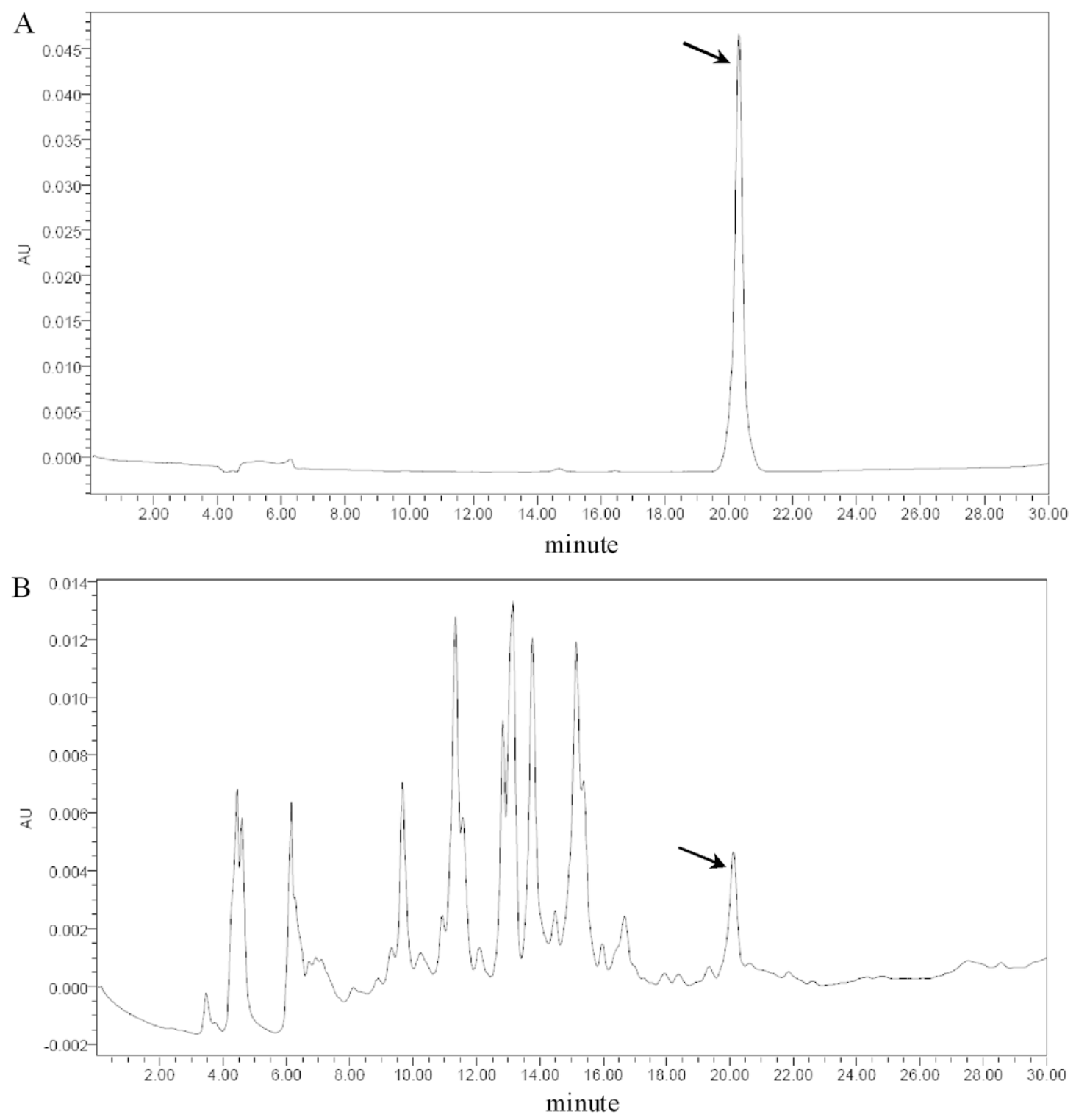

Fig. 2. Typical chromatogram of the standard solution (A) and methanol extract of cowpea (B). The arrows showed the ABA peak in both pictures (A and B) 


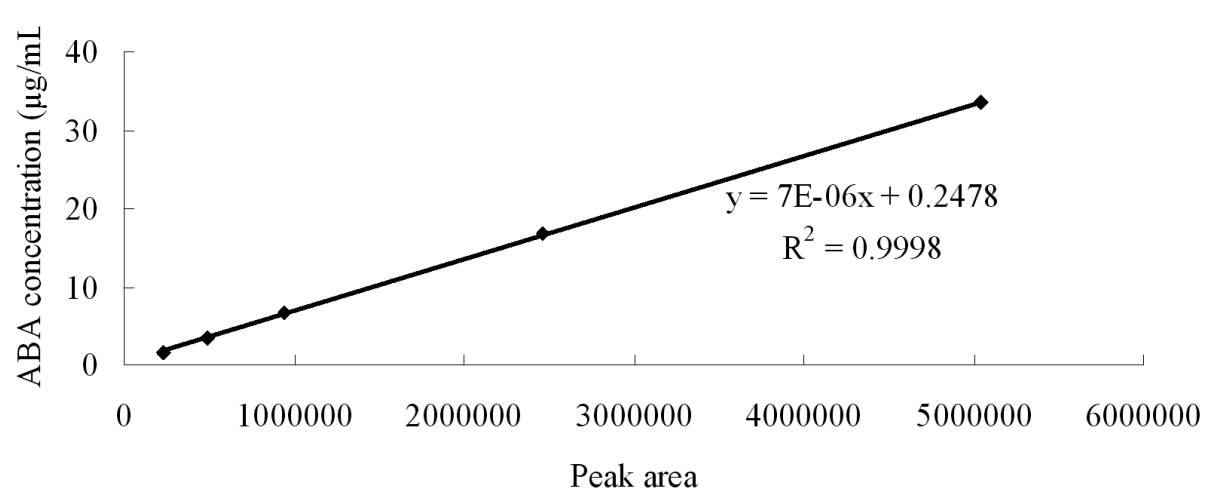

Fig. 3. Calibration curve of ABA standard solution

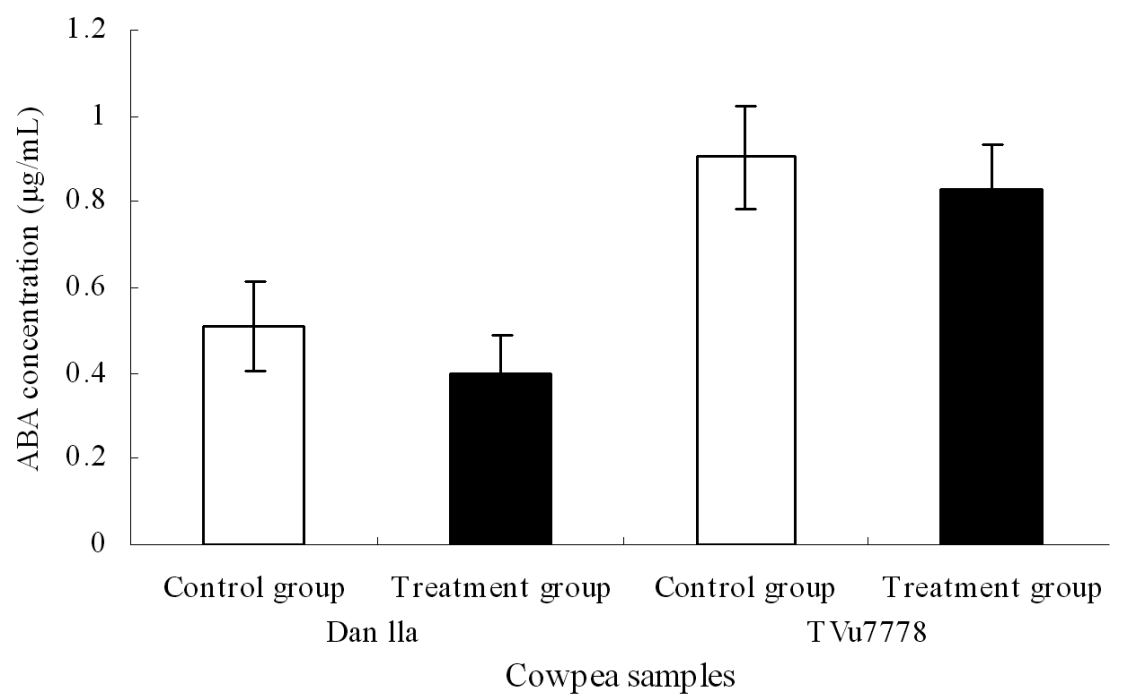

Fig. 4. Endogenous ABA contents of cowpea samples

Table 1. Validation data of the proposed HPLC method

\begin{tabular}{lc}
\hline Components & ABA \\
\hline LOD $(\mu \mathrm{g} / \mathrm{mL})(S / N=3)$ & 0.770 \\
LOQ $(\mu \mathrm{g} / \mathrm{mL})(S / N=10)$ & 2.568 \\
Stability R.S.D $(\%)$ & 2.054 \\
Precision R.S.D $(\%)$ & 1.992 \\
Recovery rate $(\%)$ & 91.56 \\
\hline
\end{tabular}

Precision was determined by assaying standard solution at $6.72 \mu \mathrm{g} \mathrm{mL} \mathrm{m}^{-1}$ for five times and R.S.D was fixed on $2.9 \%$, which demonstrated that this method is quite precise. Repeatability was tested using five independent samples (R.S.D 1.1\%) and the result indicated that this method is reproducible and able to be used widely. The stability test was performed through analyzing sample solutions at $0,2,4,6,8 \mathrm{~h}$, respectively and the R.S.D values of peak areas were more than $1 \%$, manifesting that the experimental samples can keep stable for at least $8 \mathrm{~h}$ at $4^{\circ} \mathrm{C}$. Accuracy test was carried out in terms of recovery rate, which was set out to be $99.7 \%$ with R.S.D of $1.7 \%$.

\section{Determination of $A B A$ in Cowpea}

A representative chromatogram of the methanol extracts was shown in Fig. $2 \mathrm{~B}$ and the determination quantities of every sample were illustrated in Fig. 4, which showed the endogenous ABA decreased under drought treatment and the drought sensitive line TVu7778 had higher ABA content than drought resistant variety Dan lla.

\section{Discussion}

ABA plays an important role in the signal transduction pathway of the environment stress response including drought, high salt, low temperature and so on (Liu et al., 2007; Nagamune et al., 2008). An HPLC method was successfully developed to analyze endogenous $\mathrm{ABA}$ in cowpea with a UV detector. The method was validated by some indices such as $R^{2}$, LOD, LOQ, R.S.D, which represented precision, repeatability, stability and recovery rate, respectively. 
Both LOD and LOQ were examined to estimate the sensitivity of UV detector in the present study and all of precision, repeatability and stability tests were evaluated using R.S.D value. Taken together, this developed method is acceptable, which was used to determine the endogenous ABA in all cowpea samples in this study. As indicated from these data, the HPLC method was efficacious and suitable for ABA determination and the results implying that $\mathrm{ABA}$, as a signal of drought stress response, was synthesized more to resist the stress in the non-drought-tolerant variety, alternatively, ABA biosynthesis might be limited to some extent, which is enough to deal with environmental stresses in droughtresistant plants.

\section{Conclusion}

HPLC analysis is a rational and practical analytical strategy for authentic, stable and repeatable assay of endogenous ABA in cowpea. An HPLC method was successfully developed and applied to separate and determine ABA contents in cowpea. Based on cowpea varieties with different capability of drought resistance, it is elucidated that $\mathrm{ABA}$ performs a vital function in cowpea's response and resistance to drought stress. Moreover, this assay can be effectively applied in further researches to understand the physiological and genetic mechanisms of endogenous ABA during plant development and improve plant's drought tolerance by metabolic regulation of ABA biosynthesis.

\section{Acknowledgement}

This paper was supported by Collaborative Innovation and Platform Environment Construction Projects of Guangdong Province (2015A050502049), Natural Science Foundation of Guangdong Province (2015A030313520), Foundation for Distinguished Young Talents in Higher Education of Guangdong (2013LYM 0036) and National Natural Science Foundation of China (no. 81300035 and no. 81403044).

\section{Author's Contributions}

Wei Lei: Performed the study, wrote the manuscript and/or contributed to data analysis and interpretation.

Shian Huang: Performed the study and/or contributed to data analysis and interpretation.

Shaohu Tang: Performed the study and/or wrote the manuscript.

Xiaorong Shui: Wrote the manuscript and takes full responsibility for the work as a whole, including the study design, access to data and the decision to submit and publish the manuscript.

Can Chen: Performed the study and/or contributed to data analysis and interpretation.

\section{Ethics}

This article is original and contains unpublished material. The corresponding author confirms that all of the other authors have read and approved the manuscript and no ethical issues involved.

\section{References}

Bano, A. and S. Yasmeen, 2010. Role of phytohormones under induced drought stress in wheat. Pak. J. Bot., 42: 2579-2587.

Bayuelo-Jimenez, J.S., R. Craig and J.P. Lynch, 2002. Salinity tolerance of Phaseolus species during germination and early seedling growth. Crop Sci., 42: 1584-1594. DOI: 10.2135/cropsci2002.1584

Boerjan, W., C. Genetello, M. Van Montagu and D. Inzé, 1992. A new bioassay for auxins and cytokinins. Plant Physiol., 99: 1090-1098. DOI: $10.1104 /$ pp.99.3.1090

Bressani, R., 1985. Nutritive Value of Cowpea. In: Cowpea Research, Production and Utilization, S.R. Singh and K.O. Rachie (Eds.), Books on Demand, ISBN-10: 0835760790, pp: 359-359.

Carretero, A.C. and C. Cruces-Blanco, 2004. Determination of phytohormones of environmental impact by capillary zone electrophoresis. J. Agric. Food Chem., 52: 1419-1422.

DOI: $10.1021 / \mathrm{jf0} 05377$

Chen, C., C. Tao, H. Peng and Y. Ding, 2007. Genetic analysis of salt stress responses in Asparagus Bean (Vigna unguiculata (L.) ssp. sesquipedalis Verdc.). J. Heredity, 98: 655-665. DOI: 10.1093/jhered/esm084

Ciha, A.J., M.L. Brenner and W.A. Brun, 1977. Rapid separation and quantification of abscisic acid from plant tissues using high performance liquid chromatography. Plant Physiol., 59: 821-826. DOI: $10.1104 /$ pp.59.5.821

Dobrev, P.I., L. Havlíček, M. Vágner, J. Malbeck and M. Kamínek, 2005. Purification and determination of plant hormones auxin and abscisic acid using solid phase extraction and two-dimensional high performance liquid chromatography. J. Chromatography A, 1075: 159-166. DOI: $10.1016 /$ j.chroma.2005.02.091

Du, L.M., Q.Q. Xu and Z.F. Fan, 2000. Direct determination of plant growth regulators by gas chromatography on wide bore capillary column. Chinese J. Analytical Chem., 28: 1114-1117.

Ferreira, W.D., K.G. Barbante, K.J. Elizabeth, R. Pescador and S.R. Mamoru, 2006. Thidiazuron influences the endogenous levels of cytokinins and IAA during the flowering of isolated shoots of Dendrobium. J. Plant Physiol., 163: 1126-1134. DOI: 10.1016/j.jplph.2005.07.012 
Fujita, Y., M. Fujita, K. Shinozaki and K. Yamaguchi-Shinozaki, 2011. ABA-mediated transcriptional regulation in response to osmotic stress in plants. J. Plant Res., 124: 509-525.

DOI: $10.1007 / \mathrm{s} 10265-011-0412-3$

Fukao, T., E. Yeung and J. Bailey-Serres, 2011. The submergence tolerance regulator subla mediates crosstalk between submergence and drought tolerance in rice. Plant Cell, 23: 412-427. DOI: $10.1105 /$ tpc. 110.080325

Gao, X.Q., F.J. Kong, F. Wang, H. Matsuura and T. Yoshihara, 2006. Inhibitory role of gibberellins in theobroxide-induced flowering of Pharbitis nil. J. Plant Physiol., 163: 398-404.

DOI: $10.1016 /$ j.jplph.2005.04.028

Li, J., L.T. Xiao, G.M. Zeng, G.H. Huang and G.L. Shen et al., 2005. Immunosensor for rapid detection of gibberellin acid in the rice grain. J. Agric. Food Chem., 53: 1348-1353.

DOI: $10.1021 / \mathrm{jf0487553}$

Liu, X.G., Y.L. Yue, B. Li, Y.L. Nie and W. Li et al., 2007. A G protein-coupled receptor is a plasma membrane receptor for the plant hormone abscisic acid. Science, 315: 1712-1716.

DOI: $10.1126 /$ science. 1135882

Ma, T.T., C. Huang, X.M. Meng, Q.L. Zhang and L. Zhang et al., 2011. Fingerprint analysis of Hawktea by high-performance liquid chromatography. Food Chem., 129: 551-556. DOI: 10.1016/j.foodchem.2011.04.059

Majeed, A., M. Salim, A. Bano, M. Asim and M. Hadees, 2011. Physiology and productivity of rice crop influenced by drought stress induced at different developmental stages. Afr. J. Biotechnol., 10: 5121-5136.

Martins, L.M.V., G.R. Xavier, F.W. Rangel, J.R.A. Ribeiro and M.C.P. Neves et al., 2003. Contribution of biological nitrogen fixation to cowpea: A strategy for improving grain yield in the semi-arid region of Brazil. Biol. Fertility Soils, 38: 333-339. DOI: 10.1007/s00374-003-0668-4

Munns, R., 1993. Physiological processes limiting plant growth in saline soils: Some dogmas and hypotheses. Plant Cell Environ., 16: 15-24. DOI: $10.1111 /$ j.1365-3040.1993.tb00840.x

Nagamune, K., L.M. Hicks, B. Fux, F. Brossier and E.N. Chini et al., 2008. Abscisic acid controls calcium-dependent egress and development in Toxoplasma gondii. Nature, 451: 207-210. DOI: 10.1038 /nature 06478

Paul, S., A. Kundu and A. Pal, 2011. Identification and validation of conserved microRNAs along with their differential expression in roots of Vigna unguiculata grown under salt stress. Plant Cell Tissue Organ Culture, 105: 233-242.

DOI: $10.1007 / \mathrm{s} 11240-010-9857-7$
Popelka, J.C., N. Terryn and T.J.V. Higgins, 2004. Gene technology for grain legumes: Can it contribute to the food challenge in developing countries? Plant Sci., 167: 195-206. DOI: $10.1016 /$ j.plantsci.2004.03.027

Rai, M.K., N.S. Shekhawat, G.A.K. Harish, M. Phulwaria and K. Ram et al., 2011. The role of abscisic acid in plant tissue culture: A review of recent progress. Plant Cell Tissue Organ Culture, 106: 179-190. DOI: 10.1007/s11240-011-9923-9

Ramakrishnan, K., R. Gnanam, P. Sivakumar and A. Manickam, 2005. In vitro somatic embryogenesis from cell suspension cultures of cowpea [Vigna unguiculata (L.) Walp]. Plant Cell Reports, 24: 449461. DOI: 10.1007/s00299-005-0965-5

Ross, A.R., S.J. Ambrosea, A.J. Cutlera, J.A. Feurtado and A.R. Kermode et al., 2004. Determination of endogenous and supplied deuterated abscisic acid in plant tissues by high-performance liquid chromatography-electrospray ionization tandem mass spectrometry with multiple reaction monitoring. Analytical Biochem., 329: 324-333. DOI: 10.1016/j.ab.2004.02.026

Sandhu, A.K., D.J. Gray, J. Lu and L.W. Gu, 2011. Effects of exogenous abscisic acid on antioxidant capacities, anthocyanins and flavonol contents of muscadine grape (Vitis rotundifolia) skins. Food Chem., 126: 982-988.

DOI: $10.1016 /$ j.foodchem.2010.11.105

Schwartz, S.H., X. Qin and J.A.D. Zeevaart, 2003. Elucidation of the indirect pathway of abscisic acid biosynthesis by mutants, genes and enzymes. Plant Physiol., 131: 1591-1601. DOI: $10.1104 /$ pp.102.017921

Tuteja, N., 2007. Abscisic acid and abiotic stress signaling. Plant Signal Behav., 2: 135-138.

DOI: $10.4161 /$ psb.2.3.4156

Xiong, L. and J.K. Zhu, 2003. Regulation of abscisic acid biosynthesis. Plant Physiol., 133: 29-36. DOI: $10.1104 /$ pp.103.025395

Xu, P., C.L. Liu and P. Yu, 2010. Determination of abscisic acid in fibrous root of Glycyrrhiza uralensis Fisch. by HPLC. Chinese Arch Tradit. Chinese Med., 28: 481-483.

\section{List of Symbols and Abbreviations}

Abscisic Acid, (ABA)

Gas Chromatography (GC)

Enzyme-Linked Immunosorbent Assay (ELISA)

High-Performance Liquid Chromatography (HPLC)

Limit Of Detection (LOD)

Limit Of Quantification (LOQ)

Relative Standard Deviation (RSD) 